\title{
Study on the Methods of Improving the Accuracy of Determination of Aircraft Location
}

\author{
Yongji Lu ${ }^{a}$, Ming Ming ${ }^{b}$, Kai Zhao ${ }^{c}$, Limin Song ${ }^{d}$, Lei Yuan ${ }^{e}$ \\ School of Aviation Operations and Service, Aviation University of Air Force, Changchun, 130022, China

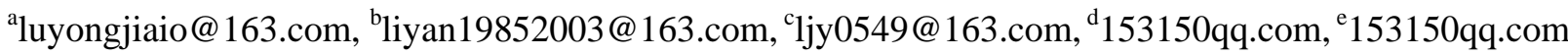

Keywords: Position of aircraft, Position Error, Positioning Measures

\begin{abstract}
The projection of the aircraft's space position on the ground at a given moment is called the aircraft's position. In the broad sky, the pilot must be located in order to fly the aircraft accurately to the intended location. The position of the aircraft is a very important job in the flight because it is a condition for the pilot to predict the flight direction and the arrival time. In flight, if you can't determine the location of the aircraft can not be sure to fly to the target heading and the advance of time, lead to no smooth completion mission, seriously accidents still can happen, and even great losses to the country and people.
\end{abstract}

\section{Determination of the Position of the Aircraft by Means of Dead Reckoning}

\subsection{Principles.}

Dead Reckoning is based on the laws of aircraft navigation, the method used to determine the position of aircraft, the time of arrival and the heading of flight, and according to the instrument instructions to maintain navigation, leading aircraft navigation. Since the track is the track of the aircraft's position, the aircraft's position must be on the track. When an aircraft remains on heading, the route drawn on the chart can be regarded as an aircraft trail. At this time, as long as the ground speed and flight time to determine the departure of a distance from a certain location, from that point on the road map from the point to the direction of the visual distance, the aircraft can be calculated position [1]. As shown in Figure 1, Point A is the starting point, according to the track $(H J)$ to determine the flight trace line, in the track line ground speed " $W$ " and flight time " $t$ " to determine the flight distance " $S$ ", so point B is the aircraft's calculated position.

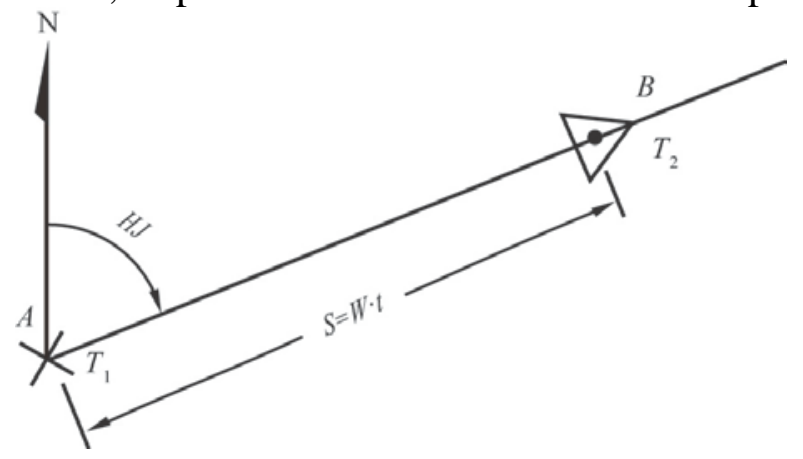

Fig. 1 Principles of aircraft Location

\subsection{Error Analysis.}

In flight, due to the change of the air wind, the errors of the instrument, as well as the pilots to maintain and calculate the uncertainty of navigation, the calculated position between the aircraft and the actual error. The distance from the calculated position is called the error of the calculated position. In addition to the above factors, the error size increases with the increase of the calculated distance [2].

As shown in Figure 2, the aircraft departed point $O$ at a certain time and reached point $M$ after a certain time. The position of the aircraft based on track, ground speed and flight time is at $M_{1} . M M_{1}$, 
then, is the position error. The angle of actual track $O M$ is the error of track $(\Delta H J)$.The difference between the actual distance $S$ and the calculated distance $S_{1}$ is the distance error $(\Delta S)$.

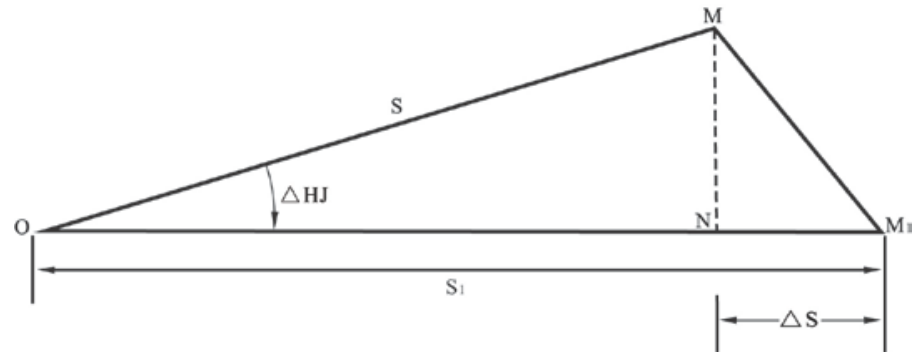

Fig. 2 Error in extrapolation position

$$
M N=\Delta H J \cdot S
$$

If the unit of the $\Delta H J$ is degree,then

$$
\begin{aligned}
M N & \approx \frac{\Delta H J}{57.3} S \\
M M_{1} & =\sqrt{\left(\frac{\Delta H J}{57.3} S\right)^{2}+(\Delta S)^{2}}=S \sqrt{\left(\frac{\Delta H J}{57.3}\right)^{2}+\left(\frac{\Delta S}{S}\right)^{2}}
\end{aligned}
$$

As can be seen from formula 3, the error of the calculated position is related to the distance(S), the track error $(\Delta H J)$ and the distance error $(\Delta S)$. The error of the track is related to the accuracy of the calculation of the heading, drift angle and the dimension angle on the chart. Therefore, the distance error depends on the error of measuring the ground speed and the error of measuring the distance on the chart [3]. A comprehensive analysis of the above shows that the error of the position is mainly due to the inaccuracy of the calculated data(track, drift angle, ground speed, wind, etc.), the inaccuracy of maintaining navigation in flight, and the inaccuracy of visual and mental calculation.

\subsection{Measures to Improve Position Accuracy.}

(1) The instruments and equipment such as the gyrocompass shall be calibrated at ordinary times to reduce instrument errors.

(2) In drawing the route, we should try to improve the accuracy of the basic data of the route and the quantity of the route.

(3) To make use of the wind direction and wind speed on the course height as recently as one hour prior to flight, calculated by the meteorological station.

(4) In flight, the specified sailing elements shall be strictly maintained, with special attention to equating the average of heading and true air speed with the predetermined value.

(5) Because the calculated position error is directly proportional to the calculated distance, the calculated distance should be shortened as far as possible and, when conditions permit, a measured position should be determined at regular intervals as a starting point for the new calculation to eliminate accumulated errors.

\section{Use of Landmarks to Locate Aircraft}

\subsection{Principles.}

The use of landmarks to determine the aircraft position is to use aerial maps to control the ground, relying on visual observation to identify landmarks to determine the aircraft position. The horizontal distance between the plane and the landmark is often used to locate the plane. The horizontal distance between the aircraft and the landmark $S_{\text {level }}$ can be determined from the aircraft's current height information and vertical viewing angle $(Z G)$.

That is 


$$
S_{\text {level }}=H \times \tan Z G
$$

Based on these relationships, the multiples of the plane's horizontal distance and height from the landmark can be determined as shown in Table 1.

Table 1 Correlation between vertical observation angle and horizontal distance

\begin{tabular}{|c|c|c|c|c|c|c|c|}
\hline$Z G$ & $45^{\circ}$ & $56^{\circ}$ & $63.5^{\circ}$ & $71.5^{\circ}$ & $76^{\circ}$ & $78.5^{\circ}$ & $80.5^{\circ}$ \\
\hline$H_{\text {times }}$ & 1 & 1.5 & 2 & 3 & 4 & 5 & 6 \\
\hline
\end{tabular}

In flight, when the vertical observation angle of the target is measured, the mental calculation can get the horizontal distance.

\subsection{Error Analysis.}

In the process of actual landmark location, the accuracy of visual measurement seriously affects the accuracy of location.

Subdivision of the formula for calculating horizontal distance:

$$
d S_{\text {level }}=\frac{\partial S}{\partial H} \cdot d H+\frac{\partial S}{\partial Z G} \cdot d Z G=\tan Z G \cdot d H+H \sec ^{2} Z G \cdot d Z G
$$

For example, when $H=4000 M, \Delta H=50 M, \Delta Z G=2^{\circ}, Z G=30^{\circ}$ :

$$
d S_{\text {level }}=\sqrt{3} / 3 \times 50+4000 \times 4 / 3 \times 2 / 57.3=215
$$

When $\mathrm{ZG}=60^{\circ}$ :

$$
d S_{\text {level }}=\sqrt{3} \times 50+4000 \times 4 \times 2 / 57.3=645
$$

Comparing the above two results, it can be seen that the smaller the $Z G$, the smaller the $d S_{\text {level, }}$, the larger the $Z G$ and the larger the $d S_{\text {level }}$.

In addition, the topographical features, seasons, day and night, meteorological conditions, visibility, and the altitude and speed of the flights in the flying area have different effects on the observation of landmarks, resulting in visual deviation.

\subsection{Methods to Improve Positioning Accuracy.}

In order to further improve the accuracy of the position of the aircraft, the following measures should be taken:

(1) Landmarks near the aircraft with small vertical viewing angles should be selected as much as possible.

(2) When judging ZG with visual force, we should practice more and improve the accuracy of visual measurement.

(3) Using different parts of the front edge of the wing to visually measure horizontal distances on the side of the landmark, the slope of the aircraft or the improper posture of the pilot can cause errors in visual measurement. Therefore, the pilot should be seated in a correct posture, the plane does not slope down to determine the position.

(4) Altimeters are designed to meet standard atmospheric conditions, with attention to the effect of temperature on altimetry indicators and to correction of temperature errors.

\section{Determination of Aircraft Position by Radio Pilotage}

There are many kinds of radio navigation equipment, such as radio compass, ultra-short wave radio, navigation station, directional station, radar, satellite navigation system and so on [3]. In flight, airspace flight or instrument flight, the pilot often uses the azimuth of the magnetoelectric station or the azimuth of the magnetic plane to determine the position of the aircraft and the position of the aircraft. If the pilot can not see the ground, can also use the radio compass to check over the distance, master the turning time, control the airspace boundary, avoid restricted areas and through 
the front entrance and exit.

\subsection{Principles of Positioning.}

The aircraft's connection to the station is called the magnetic bearing to the station, and at every moment there is such a connection between the aircraft and the station [4]. Therefore, the magnetic bearing to the station line is also known as the plane location line. The magnetic bearing to the station is a constant on the same predetermined radio bearing line.

In flight, the magnetic bearing to the station $\left(D F_{\text {指}}\right)$ indicated can be compared with $D F_{\text {预 }}$ to determine the instant entry into a predetermined bearing line [5, 6]. As shown in Figure 3. When $D F_{\text {指 }}$ is smaller than $D F_{\text {预 }}$, it means that the aircraft has not yet flown; When $D F_{\text {指 }}$ is equal to

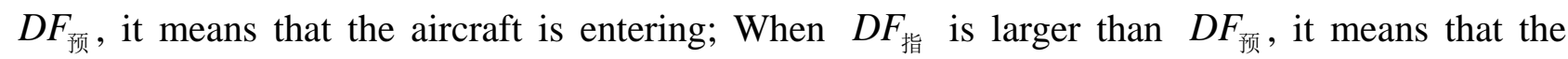
plane has flown. If the station is on the left side of the plane, the opposite is true.

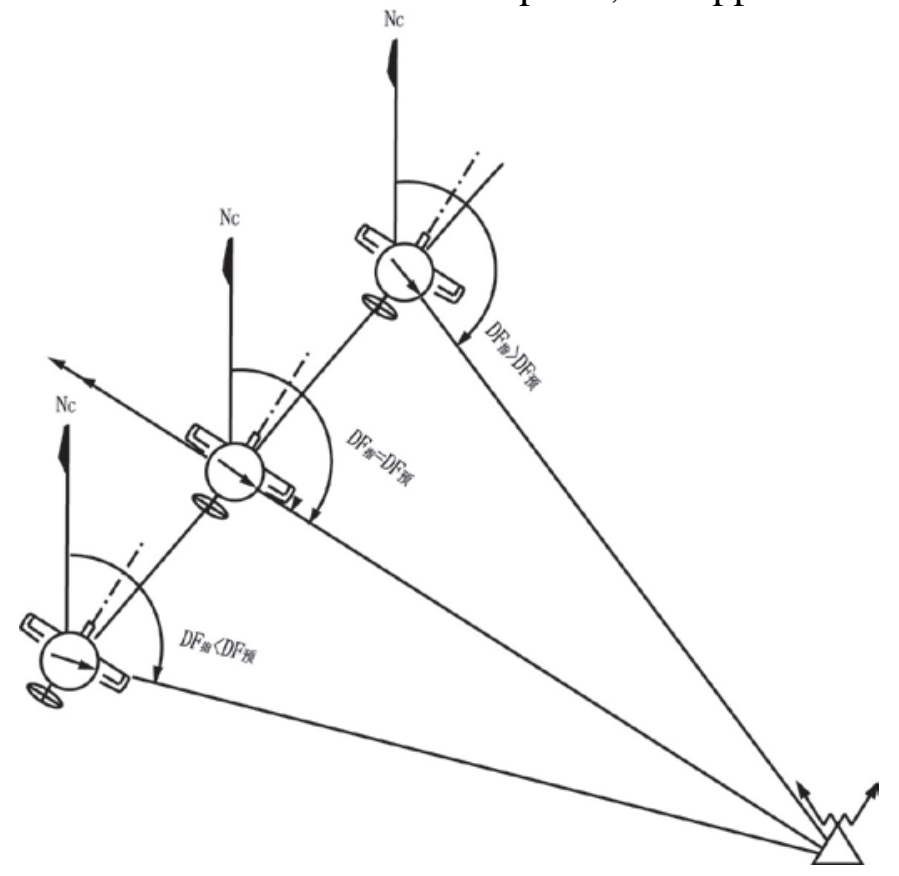

Fig. 3 Positioning Principle

\subsection{Error Analysis.}

When the radio compass is used to enter the predetermined azimuth, there are certain errors in the measured relative azimuth due to the error of the compass itself, such as maintaining the heading, tuning the radio station, reading the station's relative azimuth inaccurately and so on.

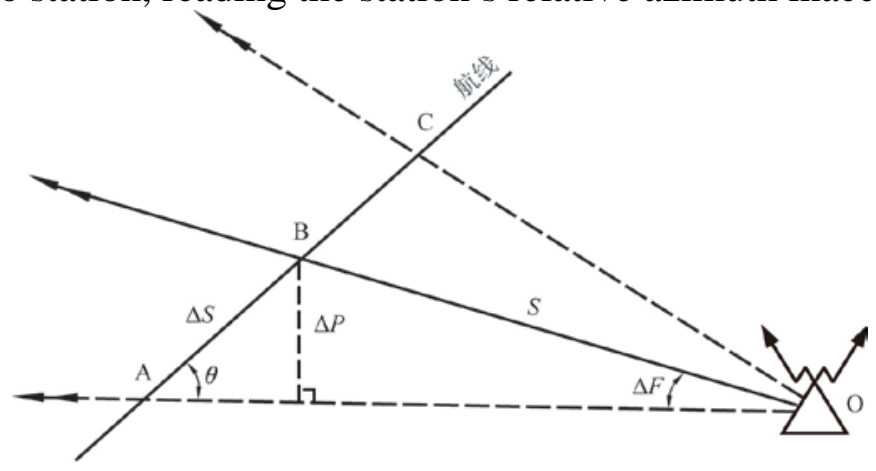

Fig. 4 Error in entering the predetermined azimuth

As shown in Figure 4, $O B$ is the predetermined azimuth line. Because of the error in azimuth $(\Delta F)$, the pilot assumes the position of the aircraft at point $\mathrm{A}$. As can be seen from the diagram, the error between position $A$ and position $B(\Delta S)$ is 


$$
\Delta S=\frac{\Delta P}{\sin \theta}
$$

In the equation, $\Delta P$ is azimuth line error; $\theta$ is the intersection angle of the same azimuth line, and

$$
\Delta P=S \cdot \sin \Delta F
$$

In which $S$ is the distance from point $B$ to station. Since the value of $\Delta F$ is generally small, usually $2^{\circ} \sim 3^{\circ}$, the $\Delta F$ in the formula is in radians or, if in degrees, the above formula is changed:

$$
\Delta P \approx \frac{S \cdot \Delta F}{57.3^{\circ}}
$$

To replace the top formula (6)

$$
\Delta S \approx \frac{S \cdot \Delta F}{57.3^{\circ} \sin \theta}
$$

Analytically, the position error $(\Delta S)$ is related to the distance of $B$ to radio station $S$, and to the angle $\theta$ of heading and azimuth.

\subsection{Measures to Improve Positioning Accuracy.}

In addition to the relative azimuth of the quasi-radio station, a radio station with a close distance, a predetermined bearing line and a track line (route) close to the vertical should be selected as far as possible. But these measures to reduce errors are sometimes contradictory to each other. In some cases, the radio stations available for selection are far away, but the angle of flight and azimuth is close to 90 degrees; sometimes the choice of radio station is close, but the route and azimuth angle is smaller. In this case, to judge the error size, we must use the formula to make a concrete calculation, and then based on the calculation results to make a choice.

\section{Trends in Positioning Methodology}

In the actual flight process, the method is usually applied synthetically. With the rapid development of computers, radar, radio, laser, infrared, low light, space positioning, automatic control and other technologies, the determination of the aircraft's position and the determination of its heading, speed and altitude will be resolved by computers and various advanced pilotage equipment, and the automation and accuracy of airborne pilotage will be increased [7]. Military positioning systems often operate in hostile environments, thus requiring them to be resistant to interference, counter-exploitation, deception and destruction. In order to prevent enemy from detecting their own operational platform by navigation signal, navigation user equipment should work passively.

In summary, the following requirements are required for future positioning: Global coverage; Be of high accuracy and do not change with the dynamics of time, place, height and carrier ;Location information, in addition to position, time, speed and heading posture ;The location information is continuous and has a high updating rate, and the public reference system can be used; Unlimited number of users and passive operation of user equipment; Anti-interference, anti-exploitation, anti-deception and anti-destruction capabilities.

\section{References}

[1] XU Zhendong, Air Navigation [M], Aviation University of Air Force, 2007.06.

[2] WEI Jingtang, Air Navigation [M], Aviation University of Air Force, 2009.12.

[3] ZHANG Huan, Air Navigation [M], Southwest Jiaotong University, 2016.08. 
[4] WANG Chunguang, WANG Xin, SUN Hang. Air Navigation Methods with the Application of Preset Azimuth Line [J]. Journal of Air Force Aviation University. 2017.12(6):36-39.

[5] LU Yongji, WANG Chunguang, WANG Xin. The application of radio azimuth in air navigation [J]. China Science and Technology Reciew. 2016.10(21):356-357.

[6] WANG Chunguang, WANG Deming, WANG Xin. Using radio azimuth to impplement air navigation method [J]. Journal of Air Force Aviation University. 2015.04(2):22-23.

[7] TAO Mei. Aeronautical Chart [M]. Beijing: Tsinghua University Press. Beijing Jiaotong University Press.2015. 